\title{
Use of a synthetic form Avrodes for modification of the genome of common wheat
}

\author{
Davoyan R.O. ${ }^{1 *}$, Bebykina I.V. ${ }^{1}$, Davoyan E.R. ${ }^{1}$, Mikov D.S. ${ }^{1}$, Zubanova Y.S. ${ }^{1}$, \\ Boldakov D.M. ${ }^{1}$, Zinchenco A.N. ${ }^{1}$, Badaeva E.D. ${ }^{2}$, Salina E.A. ${ }^{3}$, Adonina I.G. ${ }^{3}$ \\ ${ }^{1}$ National Center of Grain named after P.P. Lukyanenko, Krasnodar, Russia \\ ${ }^{2}$ Vavilov Institute of General Genetics, RAS, Moscow, Russia \\ ${ }^{3}$ Institute of Cytology and Genetics, SB RAS, Novosibirsk, Russia \\ *e-mail:davoyanro@mail.ru
}

The synthetic form Avrodes (BBAASS) was used for modification of the genome of common wheat. This form possesses the ability of Aegilops speltoides to suppress the activity of $P h$ gene(s) and stimulate homoeologous chromosome pairing. Synthetic recombinant forms (RS-forms) were obtained in which the first two genomes A and B originate from common wheat, and the third genome combining the chromosomes of the $\mathrm{S}$ genome from Aegilops speltoides with the chromosomes of other wild species. Introgression lines obtained on the basis of RS-forms can carry genetic material of two wild species, both separately and together. Currently a cytological study (C-banding and FISH) of lines derived from the synthetic recombinant form RS7 (BBAAUS) has been carried out. Lines are resistant to leaf rusts and may presumably have genetic material from both Ae. speltoides and Ae. umbellulata. Chromosomal changes affected 10 of the 12 studied lines. In most cases the lines carry translocations from Ae. speltoides. Translocations from this species were identified on chromosomes $1 \mathrm{D}, 2 \mathrm{D}, 3 \mathrm{D}, 2 \mathrm{~B}$, $4 \mathrm{~B}, 5 \mathrm{~B}$ and $7 \mathrm{~B}$. Lines with substituted chromosomes $1 \mathrm{~B}(1 \mathrm{~S}), 4 \mathrm{D}(4 \mathrm{~S}), 5 \mathrm{D}(5 \mathrm{~S})$ and $7 \mathrm{D}(7 \mathrm{~S})$ were also identified. Lines with translocations only from Ae. umbellulata not identified. Two lines carry simultaneously genetic material from Ae. speltoides and Ae. umbellulata. In line 3379 translocations were detected in the short arm of chromosome 7D from Ae. umbellulata (T7DL.7DS-7US) and on chromosomes 5B, 1D, 2D from Ae. speltoides. Line 4626 presumably has a translocation on the long arm of chromosome 2D from Ae. umbellulata (T2DS.2DL-2UL) and translocation T7SS.7SL7DL from Ae. speltoides. The translocations T1DS.1DL-1SL, T3DS.3DL-3SL from Ae. speltoides and T2DS.2DL-2UL, T7DL.7DS-7US from Ae. umbellulata obtained for the first time. 